\title{
Utility of PAX8 and PAX2 Immunohistochemistry in the Identification of Renal Cell Carcinoma in Diagnostic Cytology
}

\author{
Stewart M. Knoepp, M.D., Ph.D., Lakshmi P. Kunju, M.D., \\ and Michael H. Roh, M.D., Ph.D.
}

The diagnosis of metastatic renal cell carcinoma (RCC) in cytology specimens may be difficult to confirm on the basis of cytomorphology alone. Often, immunohistochemistry serves as an important adjunct in confirming this diagnosis. Recently, PAX2 was shown to be useful in this regard. In this study, we sought to compare the utility of PAX8 to that of PAX2 immunohistochemistry in the diagnosis of RCC in cytology specimens. First, we verified the performance of PAX8 immunohistochemistry on a tissue microarray (TMA) composed of 54 cases of RCC; PAX8 immunoreactivity was seen in at least $10 \%$ of the tumor cells in all cases. Next, we applied PAX8 immunohistochemistry to cell block sections prepared from 24 cases of $R C C$, obtained from fine-needle aspirates and effusion specimens. PAX2 immunohistochemistry was performed for comparison. Immunopositivity was defined as the presence of nuclear staining in at least $10 \%$ of tumor cell nuclei. Immunoreactivity for PAX8 and PAX2 was seen in 21 (88\%) and 20 (83\%) of the 24 cases, respectively. The presence of either PAX8 or PAX2 immunostaining was present in 22 of 24 cases, thus showing a total sensitivity of $92 \%$. Overall, the results indicate that PAX8 and PAX2 are diagnostically useful adjuncts in confirming the diagnosis of RCC in cytology specimens. Diagn. Cytopathol. 2012;40:667-672. @ 2010 Wiley Periodicals, Inc.

Key Words: PAX2; PAX8; renal cell carcinoma; cytology; fine-needle aspiration

In the United States, renal cancer accounts for approximately $4 \%$ of malignancies and is predicted to cause approximately 13,000 cancer-related deaths in $2010 .{ }^{1}$ The

Department of Pathology, University of Michigan Medical School, Ann Arbor, Michigan

*Correspondence to: Michael H. Roh, M.D., Ph.D., 2G332 UH, Department of Pathology, University of Michigan Health System, 1500 E. Medical Center Drive, Ann Arbor, Michigan 48109.

E-mail: mikro@med.umich.edu

Received 25 August 2010; Accepted 14 October 2010

DOI 10.1002/dc.21590

Published online 31 December 2010 in Wiley Online Library (wileyonlinelibrary.com). majority of renal cancers are renal cell carcinomas (RCC), a histologically diverse entity encompassing various subtypes including clear cell, papillary, chromophobe, sarcomatoid, and Xp11.2 translocation RCCs. ${ }^{2}$ Metastases secondary to RCC are usually seen in patients with known high-grade, high-stage disease (i.e., $\geq$ AJCC stage T3). However, RCC occasionally presents initially as a metastasis or malignant effusion. ${ }^{3-5}$ While RCC is an unusual source of metastatic carcinoma, it is especially important to differentiate RCC from other carcinomas as $\mathrm{RCC}$ represents one of the most aggressive genitourinary malignancies. Confirming RCC as the source of metastasis enables for appropriate pathologic staging thereby facilitating optimal therapeutic intervention. ${ }^{6}$ While RCCs exhibit distinctive cytomorphologic features in primary as well as metastatic deposits, ${ }^{7}$ the lack of perfect specificity of these features underscores the need for adjuncts such as cytogenetics and immunohistochemistry in the diagnosis and subclassification of RCCs. ${ }^{8-11}$

PAX8 and PAX2 are both members of the paired box gene (PAX) family of transcription factors that serve important roles in early metazoan development. Specifically, PAX2 has been most intensively studied in the kidney where it has been shown to play a crucial role in renal development. ${ }^{12}$ In addition, PAX8 is critical in renal and thyroid development. Recently, the expression of both has been reported in the female genital tract. ${ }^{13-16}$ Accordingly, the use of these two markers as immunohistochemical adjuncts in establishing the site of origin for carcinomas is becoming increasingly appreciated. For instance, PAX2 and PAX8 expression has been demonstrated in malignant neoplasms of Müllerian and renal origin in surgically resected specimens. ${ }^{14-23}$ Recently, the diagnostic utility of PAX8 immunohistochemistry in identifying Müllerian carcinomas in fine needle aspiration (FNA) and effusion specimens has been reported by several groups. ${ }^{24-26}$ 
There are limited reports that investigate the role of PAX2 immunohistochemistry in the diagnosis of RCC in cytology. ${ }^{19,27}$ In the reports by Gokden et al. and Wasco et al., PAX2 immunoreactivity was observed in $61 \%$ and $79 \%$ of RCCs, respectively. As there are no reports to date that examine the role of PAX8 in the cytologic diagnosis of RCC, we sought to investigate and compare the diagnostic utility of PAX8 and PAX2 in our study.

\section{Methods}

The study was approved by the University of Michigan Institutional Review Board. The electronic pathology database was searched from 2000 to 2010 for cases in which a cytologic diagnosis of RCC was rendered in patients with histologically confirmed disease based on resections of the primary renal tumor and/or biopsies of metastases. Twenty-four cases with sufficient material in the cell block preparations were selected for immunohistochemical analysis. Additionally, we utilized a tissue microarray (TMA) that consisted of 54 cases of primary RCC: 30 clear cell RCCs; 17 papillary RCCs; and 7 chromophobe RCCs.

Immunohistochemistry for PAX2 and PAX8 was performed as previously reported. ${ }^{26}$ Essentially, 4- $\mu \mathrm{m}$ unstained sections were prepared from the TMA and cell blocks, deparaffinized, and immunostained after antigen retrieval in $0.01 \mathrm{M}$ citrate buffer, $\mathrm{pH} 6.0$ (DAKO, Carpinteria, CA). Immunohistochemistry was performed using the rabbit polyclonal anti-PAX2 (1:100 dilution; Invitrogen, Camarillo, CA) and rabbit polyclonal anti-PAX8 (1:200 dilution; Protein Tech, Chicago, IL) antibodies along with appropriate controls. The EnVision+ System for use with rabbit primary antibodies (DAKO, Carpinteria, CA) was used as the secondary antibody.

TMA immunohistochemistry results for PAX8 were reviewed (LPK) and subdivided into three groups based on the proportion of cells exhibiting nuclear immunoreactivity: minimal or no positivity $(<10 \%$ of cells), focal positivity (10-50\% of cells), or diffuse positivity ( $>50 \%$ of cells). Each immunostained slide prepared from the cell blocks were reviewed (SMK and MHR) and considered positive for PAX8 or PAX2 if at least $10 \%$ of tumor cells exhibited nuclear staining.

\section{Results}

To determine the efficacy of PAX8 immunohistochemistry in detecting RCCs in histologic material, a TMA consisting of 54 RCCs was initially examined. Specifically, there were 30,17 , and 7 cases of clear cell, papillary, and chromophobe RCCs, respectively (Table I). In 53 (98\%) of 54 cases, greater than $50 \%$ of the tumor cells displayed nuclear immunoreactivity for PAX8 (Fig. 1). In the
Table I. PAX8 Immunohistochemistry of RCC on Tissue Microarray

\begin{tabular}{lcccr}
\hline & $\begin{array}{c}\text { Minimal or } \\
\text { no staining } \\
(<10 \%)\end{array}$ & $\begin{array}{c}\text { Focal } \\
\text { staining } \\
(10-50 \%)\end{array}$ & $\begin{array}{c}\text { Diffuse } \\
\text { staining } \\
(>50 \%)\end{array}$ & Total \\
\hline Clear cell RCC & 0 & 0 & 30 & 30 \\
Papillary RCC & 0 & 1 & 16 & 17 \\
Type 1 & 0 & 1 & 12 & 13 \\
Type 2 & 0 & 0 & 4 & 4 \\
Chromophobe RCC & 0 & 0 & 7 & 7 \\
Total & 0 & 1 & 53 & 54 \\
\hline
\end{tabular}

RCC, renal cell carcinoma.

remaining case of a type 1 papillary $\mathrm{RCC}$, at least $10 \%$ of the tumor cells were PAX8(+).

Next, we analyzed a series of 24 cytology specimens representing three cases of primary RCC and 21 cases of metastatic RCC. For FNA specimens of the primary renal tumors, the follow-up histologic diagnoses were clear cell RCC in two cases and papillary RCC in one case. Of the 21 cases of metastatic RCC, 17 and 4 were FNA and effusion specimens, respectively (Table II). In these cases, the associated histologic diagnoses of primary tumors and/or metastatic deposits were clear cell RCC in 12 cases, papillary RCC in three cases, papillary RCC with sarcomatoid transformation in one case, pure sarcomatoid RCC in one case, and RCC of unknown subtype in four cases.

We first examined immunohistochemistry for PAX8 in the 24 cytology cases of RCC. The results are summarized in Table II. PAX8 positivity was seen in $21(88 \%)$ of 24 cases (Fig. 2). In 17 of the 21 PAX8(+) cases, greater than $50 \%$ of the tumor cells exhibited nuclear immunoreactivity for PAX8. In the other four PAX8(+) cases, between $10 \%$ and $50 \%$ of the tumor cells were immunopositive. In the remaining three cases including two cases of clear cell RCC and one case of pure sarcomatoid RCC, all of the tumor cells were $\operatorname{PAX8}(-)$. For comparison, these cases were also immunostained for PAX2. PAX2 positivity was seen in 20 (83\%) of 24 cases (Fig. 2). In 14 of these 20 PAX2(+) cases, over $50 \%$ of the tumor cells were PAX2(+). In the other six PAX2(+) cases, at least $10 \%$ of the tumor cells were immunoreactive for PAX2. Cases that were negative for PAX2 included two cases of clear cell RCC, one case of pure sarcomatoid RCC, and one RCC of unknown subtype. In these four cases, none of the tumor cells exhibited immunoreactivity for PAX2. Overall, immunoreactivity for both PAX8 and PAX2 was seen in 19 (79\%) of 24 cases. Finally, positivity for either of the two markers was detected in $22(92 \%)$ of the 24 cases. Specifically, there were two PAX2(-) RCCs that were PAX8(+). Conversely, there was one $\mathrm{PAX} 8(-) \mathrm{RCC}$ that stained positively for PAX2. 

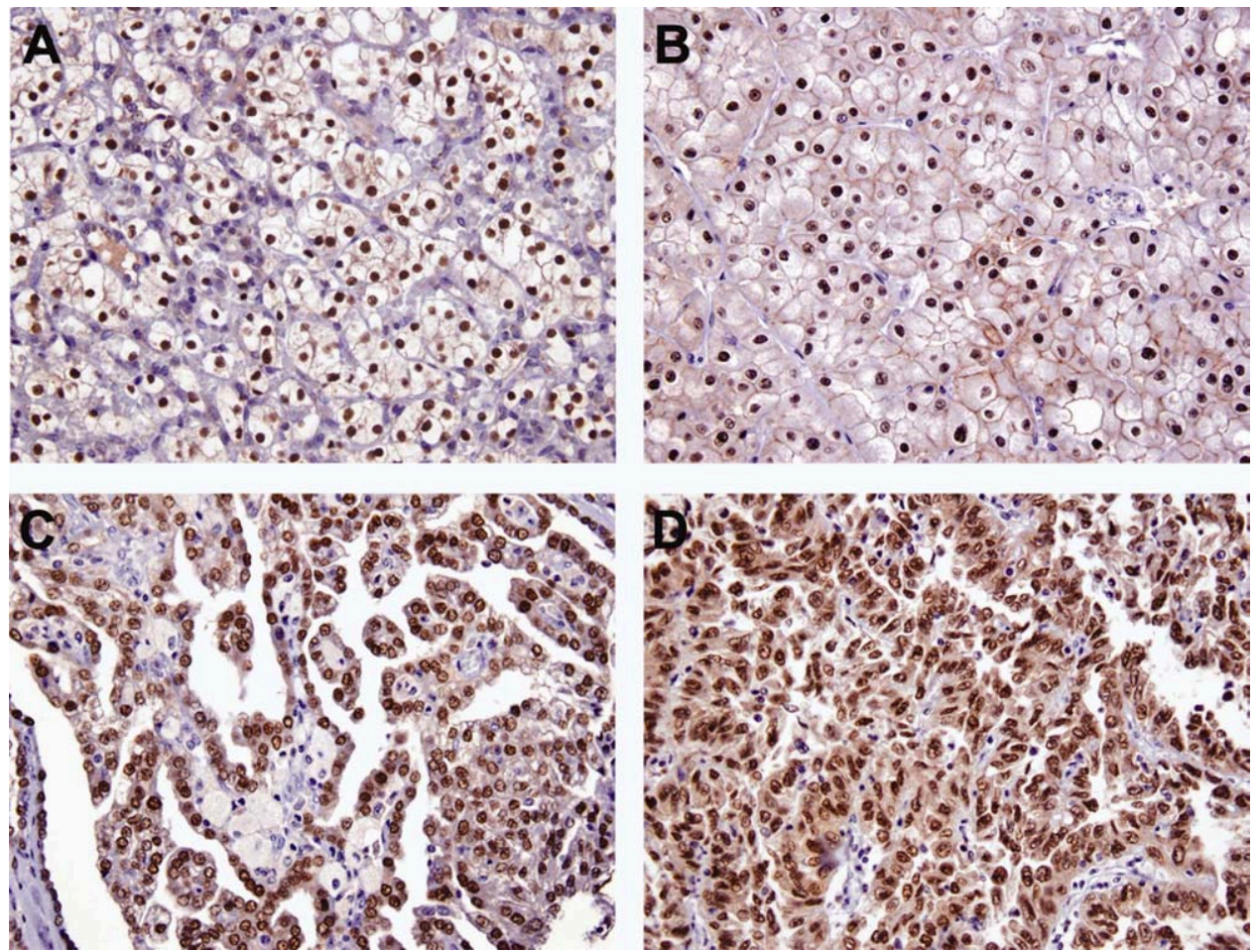

Fig. 1. PAX8 immunohistochemistry reveals nuclear staining in a various subtypes of renal cell carcinoma (RCC) on a tissue microarray (TMA). A: Clear cell RCC, $\times 400$. B: Chromophobe RCC, $\times 400$. C: Papillary RCC, type $1, \times 400$. D: Papillary RCC, type 2 , $\times 400$. [Color figure can be viewed in the online issue, which is available at wileyonlinelibrary.com.]

Table II. PAX2 and PAX8 Immunostaining in Fine-Needle Aspiration Biopsy Specimens of RCC

\begin{tabular}{lccl}
\hline FNA Site & PAX2 & PAX8 & Associated Histologic Diagnosis \\
\hline Hilar mass & - & - & Clear cell RCC \\
Subcarinal mass & + & + & Clear cell RCC \\
Paratracheal LN & + & + & Papillary and sarcomatoid RCC \\
Paratracheal LN & + & + & Clear cell RCC \\
Left renal mass & + & + & Clear cell RCC \\
Pericardial fluid & + & + & Papillary RCC \\
Pancreatic tail & - & - & Sarcomatoid RCC \\
Right renal mass & + & + & Clear cell RCC \\
Right renal mass & + & + & Papillary RCC \\
Pancreatic mass & + & + & Clear cell RCC \\
Paratracheal LN & + & + & Clear cell RCC \\
Para-renal LN & + & + & Clear cell RCC \\
Pancreas & + & + & RCC, unknown subtype \\
Adrenal & + & + & Clear cell RCC \\
Thyroid & + & + & Clear cell RCC \\
Right neck & + & + & Clear cell RCC \\
Pleural fluid & - & + & RCC, unknown subtype \\
Left neck & + & + & Papillary RCC \\
Lung, LUL & + & + & Clear cell RCC \\
Vertebral mass & + & + & RCC, unknown subtype \\
Left peri-aortic LN & + & - & Clear cell RCC \\
Ascitic fluid & + & + & RCC, unknown subtype \\
Right C-2 mass & + & + & Papillary RCC \\
Pleural fluid & - & + & Clear cell RCC
\end{tabular}

RCC, renal cell carcinoma; LUL, left upper lobe; LN, lymph node.

\section{Discussion}

PAX8 and PAX2 play important roles as transcription factors during the organogenesis of the kidney. ${ }^{12}$ In the adult kidney, nuclear expression of PAX8 has been demonstrated by immunohistochemistry in the parietal cells of Bowman's capsule and all segments of the renal tubules. $^{23}$ This expression profile partially overlaps with that of PAX2 in non-neoplastic renal parenchyma; nuclear immunoreactivity has been demonstrated in the parietal cells, distal convoluted tubules, loops of Henle, and collecting duct. ${ }^{28}$ Furthermore, in these reports, PAX8 and PAX2 expression was shown to be maintained in carcinomas derived from the renal tubule epithelium, specifically RCCs. As there are no studies to date that have investigated the use of PAX8 immunohistochemistry in metastatic renal neoplasms in diagnostic cytology, we sought to test our hypothesis that PAX8 immunostaining could serve as a useful adjunct in the diagnosis of RCCs in cytology specimens.

We first validated the use of PAX8 immunohistochemistry on a TMA composed of 54 primary RCCs and found that PAX8 was expressed all of the RCCs tested, specifically, those of the clear cell, papillary, and chromophobe subtypes. This is in agreement with the findings reported by Tong et al., who detected PAX8 expression in a high proportion of clear cell, papillary, and chromophobe RCCs. $^{23}$

We next examined PAX8 expression in 24 cytology specimens and demonstrated that the majority $(88 \%)$ of RCCs exhibited positive immunostaining for PAX8. The 

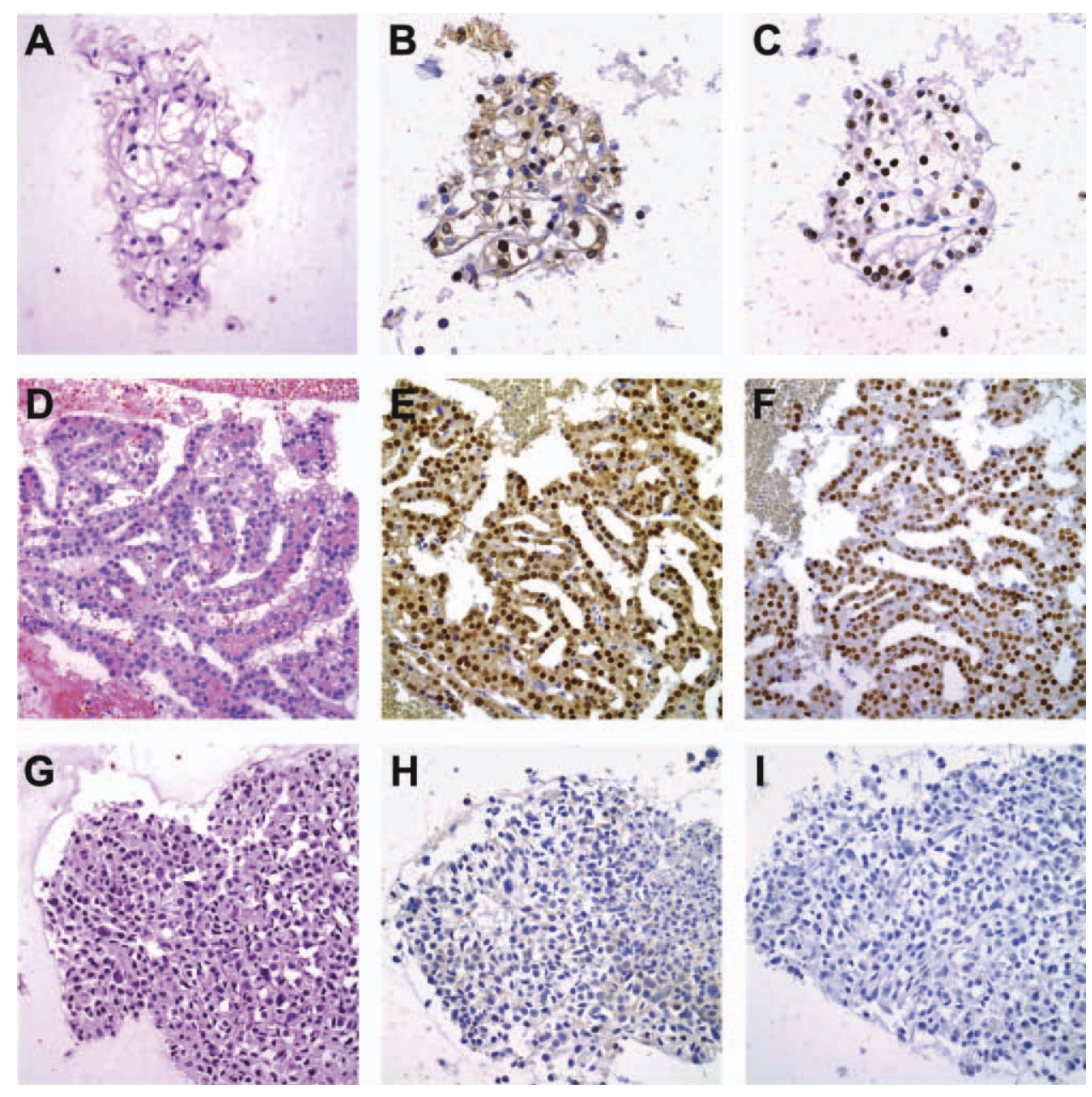

Fig. 2. PAX8 and PAX2 immunohistochemistry reveals nuclear staining in the majority of RCCs in cell block preparations from cytology specimens. A-C: Clear cell RCC, $\times 600$. D-F: Papillary RCC, $\times 400$. G-I: Sarcomatoid RCC, $\times 400$. Hematoxylin and eosin stained sections are shown in A, D, and G. PAX8 immunostains are displayed in B, E, and H. PAX2 immunostains are shown in C, F, and I. Nuclear immunoreactivity for both markers are observed in the clear cell and papillary RCC (B, C, E, F) but not in the case of sarcomatoid RCC (H, I). [Color figure can be viewed in the online issue, which is available at wileyonlinelibrary.com.]

majority of these were metastases; therefore, PAX8 can serve as a valuable immunohistochemical adjunct in confirming a metastatic tumor as RCC. Of note, one of the metastatic RCC cases in our cohort involved the thyroid gland. Caution should be exercised in interpreting PAX8 immunohistochemistry in this setting as non-neoplastic and neoplastic thyroid follicular cells are often positive for PAX8. In contrast, as thyroid follicular cells do not display immunoreactivity for PAX2, ${ }^{29}$ this marker would serve to discriminate metastatic RCC from epithelial cells of thyroid origin. In addition, three of the 24 cytology cases were FNAs of the primary renal tumor. In this setting, caution should also be exercised in interpreting the PAX8 immunostain as PAX8 immunoreactivity can be seen in benign renal tubular cells and in tumor cells. $^{23}$

Next, for comparison, we also examined our cytology case cohort for PAX2 expression. We found that 20 of the 24 cytology cases of RCC were positive for PAX2 for an overall sensitivity of $83 \%$ comparable to that for PAX8. This is concordant with the results published by Wasco et al. who demonstrated a 79\% PAX2 positivity rate in RCCs. ${ }^{27}$ Another study by Gokden et al. reported a lower PAX2 positivity rate of $61 \%$ in RCCs. ${ }^{19}$ This underscores the notion that PAX8 represents a useful additional marker to confirm a metastatic RCC, especially in the scenario where PAX2 fails to highlight the tumor cells.

Overall, the data stemming from our cytology case cohort compare similarly to a prior study examining the utility of PAX8 immunohistochemistry in the diagnosis of primary or metastatic RCCs in surgically resected specimens. ${ }^{23}$ Our results indicate that PAX8 is as sensitive as or more sensitive than other commonly used markers in confirming a diagnosis of RCC. CD10 is positive in $82 \%$ of clear cell RCCs and $59 \%$ of papillary $\mathrm{RCCs}^{30}$ which 
falls below our observed sensitivity for PAX8 (88\%). Likewise, EMA shows lower sensitivity for clear cell RCC or papillary RCC (77\% and $67 \%$, respectively). ${ }^{30}$ Wasco and $\mathrm{Pu}$ reported a sensitivity of $56 \%$ for RCC antigen and $73 \%$ for gamma-H2AX antibodies for detecting RCC in cytology specimens. ${ }^{27,31}$ Furthermore, RCC antigen shows highest sensitivity for papillary RCCs (95\%), but shows only $72 \%$ sensitivity for clear cell RCC, the most common subtype. ${ }^{32}$

The specificity for PAX8 and PAX2 in diagnostic cytology has been previously reported based on studies that examined metastatic carcinomas in predominantly effusion specimens and a limited number of FNAs. Importantly, neither PAX8 nor PAX2 expression has been observed in metastatic adenocarcinomas from lung, breast, pancreaticobiliary, and gastrointestinal tract primaries. ${ }^{19,25,26}$ Nonetheless, it should be noted that PAX8 and PAX2 expression is not completely specific for RCC. Apart from RCC, PAX8 immunoreactivity can be observed in carcinomas of Müllerian and thyroid origin. $^{24-26}$ Of note, Müllerian papillary serous carcinoma and papillary thyroid carcinoma can mimic papillary RCC due to their characteristic papillary architectural features. Because all three entities are often PAX8(+) and PAX2 expression can be seen in a subset of serous carcinomas, ${ }^{26}$ neither PAX8 nor PAX2 would be specific for RCC in this context. To distinguish between these three entities, additional immunohistochemical stains would provide valuable diagnostic aid. Specifically, Müllerian papillary serous carcinoma would be expected to be immunoreactive for one or more of the following: WT-1; ER; and PR. In contrast, papillary thyroid carcinomas and RCCs would be negative for these markers. In addition, thyroglobulin and TTF-1 would serve to differentiate papillary thyroid carcinoma from serous carcinoma and RCC. Next, Müllerian clear cell carcinomas can mimic clear cell RCCs owing to the delicate, vacuolated cytoplasm and prominent nucleoli frequently observed in both. As PAX8 and PAX2 expression can be seen in a subset of the former, ${ }^{13,18}$ these markers would not be helpful in differentiating clear cell RCC from Müllerian clear cell carcinoma. Again, additional immunohistochemical markers such as cytokeratin 7 (CK7) and RCC antigen can be helpful in this regard. ${ }^{18}$ Specifically, a CK7(-) immunophenotype would favor clear cell RCC whereas CK7-positivity would be more consistent with a Müllerian clear cell carcinoma. Furthermore, demonstrating immunoreactivity for RCC antigen would support a diagnosis of RCC.

In conclusion, our study demonstrates that both PAX8 and PAX2 are useful markers for identifying metastatic RCC in cytology specimens. While PAX8 shows a slight increase in sensitivity over PAX2 in RCCs, the highest sensitivity and therefore greatest utility lies in using both markers for confirming a diagnosis of RCC.

\section{Acknowledgments}

The authors would like to thank Nancy McAnsh and Alan Burgess in the University of Michigan Comprehensive Cancer Center Research Histology and Immunoperoxidase Laboratory for their technical expertise and assistance in performing immunohistochemistry.

\section{References}

1. Jemal A, Siegel R, Xu J, Ward E. Cancer Statistics, 2010. CA Cancer J Clin 2010;60:277-300.

2. Murphy W, Grignon DJ, Perlman EJ. Tumors of the kidney, bladder, and related urinary structures. In: Steven G, Silverberg M, editors. Washington, DC: American Registry of Pathology; 2004. $394 \mathrm{p}$.

3. Ohnishi H, Abe M, Hamada H, et al. Metastatic renal cell carcinoma presenting as multiple pleural tumours. Respirology 2005;10: 128-131.

4. Singla R, Bhattacharya D, Chhabra SK, Menon MP. Pleural effusion in renal cell carcinoma-A rare presenting feature. Indian $\mathrm{J}$ Chest Dis Allied Sci 1987;29:29-35.

5. Ellis CL, Burroughs F, Michael CW, Li QK. Cytology of metastatic renal medullary carcinoma in pleural effusion: A study of two cases. Diagn Cytopathol 2009;37:843-848.

6. Pavlidis N, Briasoulis E, Hainsworth J, Greco FA. Diagnostic and therapeutic management of cancer of an unknown primary. Eur $\mathrm{J}$ Cancer 2003;39:1990-2005.

7. Truong LD, Todd TD, Dhurandhar B, Ramzy I. Fine-needle aspiration of renal masses in adults: Analysis of results and diagnostic problems in 108 cases. Diagn Cytopathol 1999;20: 339-349.

8. Kunju LP, Wojno K, Wolf JS, Jr, Cheng L, Shah RB. Papillary renal cell carcinoma with oncocytic cells and nonoverlapping low grade nuclei: Expanding the morphologic spectrum with emphasis on clinicopathologic, immunohistochemical and molecular features. Hum Pathol 2008;39:96-101.

9. Leroy X, Farine MO, Buob D, Wacrenier A, Copin MC. Diagnostic value of cytokeratin 7. CD10 and mesothelin in distinguishing ovarian clear cell carcinoma from metastasis of renal clear cell carcinoma. Histopathology 2007;51:874-876.

10. Roh MH, Dal Cin P, Silverman SG, Cibas ES. The application of cytogenetics and fluorescence in situ hybridization to fine-needle aspiration in the diagnosis and subclassification of renal neoplasms. Cancer Cytopathol 2010;118:137-145.

11. Wu A, Kunju LP, Cheng L, Shah RB. Renal cell carcinoma in children and young adults: Analysis of clinicopathological, immunohistochemical and molecular characteristics with an emphasis on the spectrum of Xp11.2 translocation-associated and unusual clear cell subtypes. Histopathology 2008;53:533-544.

12. Dressler GR. The specification and maintenance of renal cell types by epigenetic factors. Organogenesis 2009;5:73-82.

13. Bowen NJ, Logani S, Dickerson EB, et al. Emerging roles for PAX8 in ovarian cancer and endosalpingeal development. Gynecol Oncol 2007; 104:331-337.

14. Roh MH, Kindelberger D, Crum CP. Serous tubal intraepithelial carcinoma and the dominant ovarian mass clues to serous tumor origin? Am J Surg Pathol 2009;33:376-383.

15. Tong GX, Chiriboga L, Hamele-Bena D, Borczuk AC. Expression of PAX2 in papillary serous carcinoma of the ovary: Immunohistochemical evidence of fallopian tube or secondary Mullerian system origin? Mod Pathol 2007;20:856-863.

16. Tung CS, Mok SC, Tsang YT, et al. PAX2 expression in low malignant potential ovarian tumors and low-grade ovarian serous carcinomas. Mod Pathol 2009;22:1243-1250. 
17. Chivukula M, Dabbs DJ, O'Connor S, Bhargava R. PAX 2: A novel Mullerian marker for serous papillary carcinomas to differentiate from micropapillary breast carcinoma. Int $\mathrm{J}$ Gynecol Pathol 2009;28:570-578.

18. Gokden N, Gokden M, Phan DC, McKenney JK. The utility of PAX-2 in distinguishing metastatic clear cell renal cell carcinoma from its morphologic mimics: An immunohistochemical study with comparison to renal cell carcinoma marker. Am J Surg Pathol 2008;32:1462-1467.

19. Gokden N, Kemp SA, Gokden M. The utility of Pax-2 as an immunohistochemical marker for renal cell carcinoma in cytopathology. Diagn Cytopathol 2008;36:473-477.

20. Tong GX, Melamed J, Mansukhani M, et al. PAX2: A reliable marker for nephrogenic adenoma. Mod Pathol 2006;19:356-363.

21. Albadine R, Schultz L, Illei P, et al. PAX8 (+)/p63(-) immunostaining pattern in renal collecting duct carcinoma (CDC): A useful immunoprofile in the differential diagnosis of CDC versus urothelial carcinoma of upper urinary tract. Am J Surg Pathol 2010;34:965969.

22. Laury AR, Hornick JL, Perets R, et al. PAX8 reliably distinguishes ovarian serous tumors from malignant mesothelioma. Am J Surg Pathol 2010;34:627-635.

23. Tong GX, Yu WM, Beaubier NT, et al. Expression of PAX8 in normal and neoplastic renal tissues: An immunohistochemical study. Mod Pathol 2009;22:1218-1227.

24. McKnight R, Cohen C, Siddiqui MT. Utility of paired box gene 8 (PAX8) expression in fluid and fine-needle aspiration cytology: An immunohistochemical study of Metastatic ovarian serous carcinoma. Cancer Cytopathol 2010;118:298-302.
25. Tong GX, Devaraj K, Hamele-Bena D, et al. PAX8: A marker for carcinoma of Mullerian origin in serous effusions. Diagn Cytopathol 2010 [e-pub ahead of print].

26. Wiseman W, Michael CW, Roh MH. Diagnostic utility of PAX8 and PAX2 immunohistochemistry in the identification of metastatic Mullerian carcinoma in effusions. Diagn Cytopathol 2010 [e-pub ahead of print].

27. Wasco MJ, Pu RT. Comparison of PAX-2. RCC antigen, and antiphosphorylated $\mathrm{H} 2 \mathrm{AX}$ antibody (gamma-H2AX) in diagnosing metastatic renal cell carcinoma by fine-needle aspiration. Diagn Cytopathol 2008;36:568-573.

28. Ozcan A, Zhai J, Hamilton C, et al. PAX-2 in the diagnosis of primary renal tumors: Immunohistochemical comparison with renal cell carcinoma marker antigen and kidney-specific cadherin. Am J Clin Pathol 2009;131:393-404.

29. Zhai QJ, Ozcan A, Hamilton C, et al. PAX-2 expression in nonneoplastic, primary neoplastic, and metastatic neoplastic tissue: A comprehensive immunohistochemical study. Appl Immunohistochem Mol Morphol 2010;18:323-332.

30. Pan CC, Chen PC, Ho DM. The diagnostic utility of MOC31. BerEP4, RCC marker and CD10 in the classification of renal cell carcinoma and renal oncocytoma: An immunohistochemical analysis of 328 cases Histopathology 2004;45:452-459.

31. Wasco MJ, Pu RT. Utility of antiphosphorylated H2AX antibody (gamma-H2AX) in diagnosing metastatic renal cell carcinoma. Appl Immunohistochem Mol Morphol 2008;16:349-356.

32. Bakshi N, Kunju LP, Giordano T, Shah RB. Expression of renal cell carcinoma antigen (RCC) in renal epithelial and nonrenal tumors: Diagnostic Implications. Appl Immunohistochem Mol Morphol 2007; 15:310-315. 